\title{
AN APPROACH FOR ADAPTIVE MODEL PERFORMANCE VALIDATION WITHIN DIGITAL TWINNING
}

\author{
MADHU SUDAN SAPKOTA ${ }^{1}$, EDWARD APEH ${ }^{1}$, MARK HADFIELD ${ }^{1}$, ROYA HARATIAN ${ }^{1}$, \\ ROBERT ADEY ${ }^{2} \&$ JOHN BAYNHAM ${ }^{2}$ \\ ${ }^{1}$ Faculty of Science and Technology, Bournemouth University Poole, United Kingdom. \\ ${ }^{2}$ Wessex Institute of Technology, Ashurst Lodge, United Kingdom.
}

\begin{abstract}
The validation of the operationality of models is considered a crucial step in the model development process. Recent developments in Digital Twinning (DT) enable the online availability of operational data from the physical asset required for operational validation. The benefits of DT in situations where operational validation has formed a basis for model adaptation has also been demonstrated. However, these benefits within DT have not been fully utilized due to the lack of an approach for benchmarking the required quantity, quality and diversity of validation data and performance metrics for online model validation and adaptation. Therefore, there is a need for a framework for benchmarking validation data and metrics requirements during model validation in different domains. An approach for benchmarking the required quantity, quality and variability of validation data and performance metric(s) for online model adaptation within DT is proposed. The approach is focused on addressing the problem of parameter(s) uncertainty of a predictive model within its uncertainty boundary. It involves generating virtual test models, a primary and another reference model for the performance evaluation of one compared to the another with the benchmarked validating data and metrics within DT. This process is repeated until the dataset and/or metric(s) are promising enough to validate primary model against the reference model. The proposed approach is demonstrated using BEASY - a simulator designed to predict protection provided by a cathodic protection system to an asset. In this case, a marine structure is the focus of the study, where the protection potentials to prevent corrosion are predicted over the life of the structure. The algorithm(s) for the approach are provided within a Scientific Software (MATLAB) and integrated to the simulator-based cathodic-protection model.
\end{abstract}

Keywords: Adaptive Simulation Validation, Cathodic-Protection Digital Twin, Validating Framework.

\section{INTRODUCTION}

Simulation models that emulate entity/structure/system behaviour are widely used for prognosis and consequently to aid in Structural Health Management. While relying upon such predictive models for structural health assessment of any asset, the success of ultimate decision making depends upon the robustness of the predictive simulations. Thus, validation of the model for its applicability within the accepted performance range is a crucial aspect during simulation model development, among several iterative phases [1].

When pre-validated conceptual models that sufficiently represent the behavioural process of the system are available in the specific domain, it is preferably adopted rather than building model from zero. Parametric models built based on the pre-accepted conceptual models are then calibrated to represent the real physical asset. Calibration is made by finding the best fit of parameters values that correlates the model output to the available data from physical asset [2]. However, in multiple domains, the model requires re-calibration when parameters value 
varies with time. This tends to mainly be due to the complex and non-uniform change in the material properties of the asset in response to the environmental factors[3]. The validation of the operationality of models, therefore, also plays a role during the operational period, that is, beyond the initial model building phase.

Different relevant validation techniques are used to establish the credibility of the model(s) at different stages of model development and operation in different domains [4]. Cost and resource requirements in terms of data requirement are other considerable factors for model validation but not as well understood as they need to be because of lack of meaningful information in the modelling communities [5]. Therefore, the choice of relevant validation techniques and data dependency is hugely reliant on involved domain-related expertise at best. Moreover, the entire recurring validation and calibration process demands automation along with benchmarked validation resource requirements to ensure efficiency and consistency.

Recent research has demonstrated the benefits of Digital Twins (DT) provided with simulation capability situations where adaptive simulation is necessary [6, 7]. However, due to lack of standardisation of DT concept [8], the pre-mentioned validation-related limitations persist even utilising the DT concept. The limitations include resources benchmarking for DT on representing physical asset and to validate the performance of its predictive capability, and frequent expertise requirement for performance evaluation.

The goal of this paper is to provide the approach for benchmarking the required quantity, quality and diversity of validation data and performance metrics for online model validation within DT.

The benchmarking approach will support to generate a best suitable model adaptation route that can later be implemented during the parametric adaptation of the model during realisation of virtual replica of a physical asset. The rest of the paper is structured as follows: Section 2 provides overview of the related work. Section 3 provides the proposed approach of online model validation within DT. The test result and analysis are presented in the Section 4. Finally, Section 5 comprises of the conclusion.

\section{BACKGROUND OF ADAPTIVE MODEL VALIDATION}

\subsection{Adaptive Simulation Model and Validation Assessment}

In adaptive modelling, models are built with usage/adoption of parametric models that have been validated to the level of their conceptual modelling [9]. The parametric model building in multiple domains is already facilitated by the availability of commercial process simulators (simulating software). Such simulators are generally based on current conceptual behavioural understanding of system/asset, often involving large systems of differential equations, and they are implemented in complex computer programs [10].

Usually model building consists of two major re-iterative tasks: i) determining and addressing uncertainty that typically involves updating the parameters to the computer simulator and ii) accounting for inadequate physics of the model. When predefined simulators that have been verified and validated are adopted, the re-iterative task is mostly focused towards the first task [11] for the convergence of model performance to the real system/asset's behaviour.

Among, different methods used to validate simulation models, operational validation is widely applied at the last stage of model building, that is, during realisation of virtual replica of a physical asset [1]. It is performed by determining the error and/or uncertainty of the model's prediction performing the comparative analysis between the predicted and corresponding 
physical system-related data. The operational validation data (information) requirements might vary due to the behavioural complexity of the physical system [5]. This appears as an increase in cost, time and risk of wrong assumption due to shortage of resources, that is, the data or information required. Uncertainty in the validating measurement data, in most of the cases, is irreducible. Therefore, benchmarking the data to their quantitative, qualitative and diversity requirement to track the model's performance is essential to avoid the wrong assumption about model's performance.

The measurement of accuracy between model outputs and real data is made using performance criteria [12]. To select appropriate metric (performance criteria) on model operational validation assessment, the identification of the model's nature, that is, deterministic or stochastic, provides first step [4]. Moreover, the processes of validation and adaptation/calibration are deeply connected, and the methodology adopted to coordinate these two processes is also important [13]. While deterministic approaches are preferred for model adaptation, that is, parameter updating [14], it is suggested to use deterministic criterion for validation as well as during model adaptation.

\subsection{DT and Simulation Model Validation Assessment}

DT is gaining popularity in modelling and simulation field during recent years. DT can be defined as a virtual instance of a physical system (twin) that is continually updated with the latter's performance, maintenance and health status data throughout the physical system's life cycle [6]. Implementing DT concept on first instance means online availability of necessary physical asset-related required data for decision making. Though, DT has achieved some maturity in conceptual level, it is still at the infancy on its application, and only a few studies have specifically discussed the methods for its construction and implementation [8]. Nevertheless, there is no doubt that the most important aspect of DT appeared since the very first interpretation of the DT concept is 'an integrated online simulation' $[15,16]$. Therefore, validation of DT or DT-based simulation model is still relevant from model building perspective even while adopting DT concept for predictive simulation.

Artificial intelligence is one of the features recommended for DT on self-performing the analytical tasks including simulation performance validation and adaptation [8, 17]. However, lack of proper framework persists for continuous self-improvement [18] of DT as hindrance to enable intelligence capacity within DT.

While focus on the requirement of online calibration/adaptation has begun to receive significant attention in the literatures, the online model validating framework within DT seems to be left out.

\section{APPROACH FOR BENCHMARKING ONLINE OPERATIONAL MODEL VALIDATION REQUIREMENTS}

Tailoring any parametric simulation model to represent the physical system requires a suitable model adaptation route $[19,20]$. The route is anticipated with benchmarked fewer possible validating data dependency to reduce cost and the requirement for iteration when updating parameters. With the goal to provide support on benchmarking of the resource requirements for model validation during continuous calibration/ adaptation, the approach is proposed.

The approach for benchmarking the model operational validation requirements is supported with multiple reference virtual experiments (Fig. 1). Each reference experiment consists of two different virtual simulation models (not replica of the physical asset) with similar performances but with different response output data. First model is considered as 
the primary model to be validated and updated/calibrated, while another model is used as the reference model (3.1), to which the first model should converge.

Only if convergence of initial primary model to the acceptance range of reference model is reached in reference experiments, the validation requirements are accepted after re-testing in similar other reference experiments.

Then, the accepted model validating data and/or metric can be used as the benchmark of data required from real asset and performance validating metric, respectively, at the time of realisation of the virtual replica.

Adopting DT concept and automation of the approach can be achieved by leveraging the analytical support from the DT.

\subsection{Virtual Reference Model Generation}

The performance of validation data and metric provided/selected/benchmarked during model validation and adaptation can be analysed either comparing the adapted/calibrated model with higher precision model or with virtual prototype. Using the virtual prototype as a reference model in the virtual testing is the key not only to reduce of the cost and effort, but also to avoid inconvenience for hardware tests.

While at this stage, the research is focused towards assessing the parametric uncertainties of the model, the reference model is generated with change in the parameter(s) values to the initial primary model but within reasonable range(s). Automated analytical support within DT can generate such virtual models; however, the external support might be required to limit the parameters within reasonable ranges.

\subsection{Validation Data Determination}

For the process of model operational validation, multiple data types could be available, data could have error and uncertainty, and similar data could be generated in different inputs for

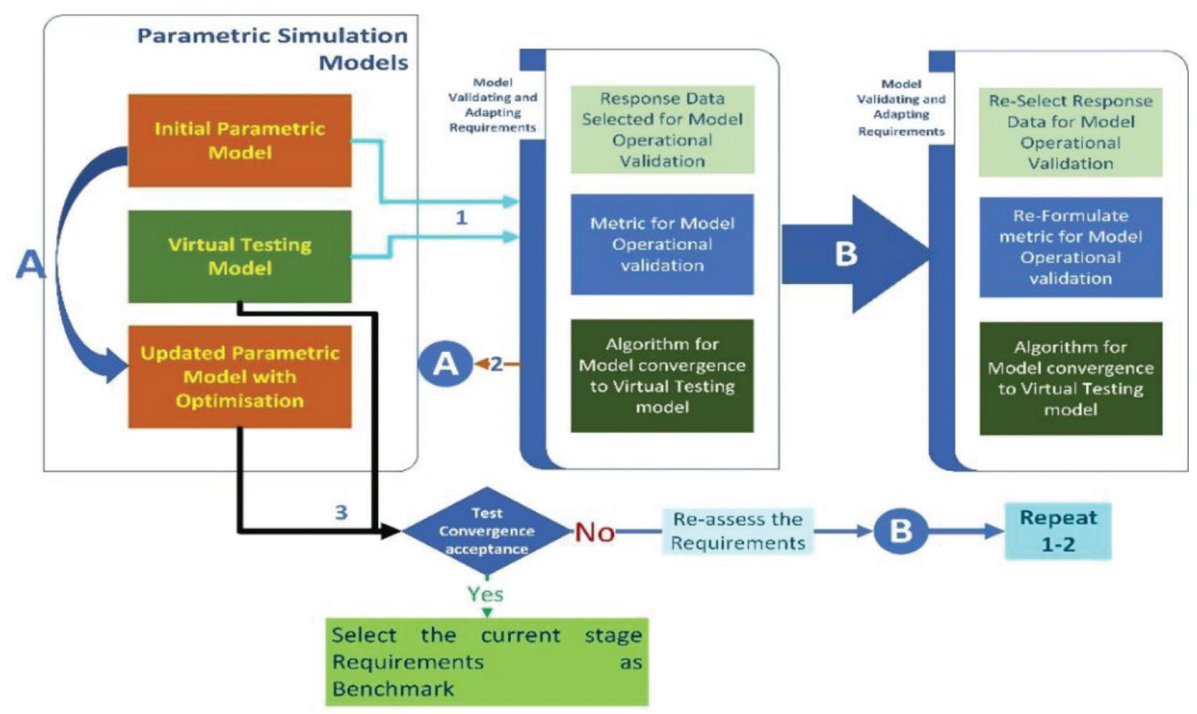

Figure 1: Illustration of reference experiment for Benchmarking Operational Validation Requirements 
simulation run. For example, in the case of cathodic-protection model validation, the data dependency ranges from surface-potential, normal current density and potential gradient-related data [21]. Increasing complexity with the growth of degree of freedoms in any simulation model also puts a strain on validation and calibration of the model. Thus, establishing proper benchmark of the data to their quantitative, qualitative and diversity requirement to track the model's performance is essential.

Data sensitivity tracking for corresponding parameters, that is, data mapping to the parameters is usually achieved by sensitivity analysis and requires no reference model. Then, fixing other involved model validating and updating attributes (e.g. metric, optimisation algorithm), the benchmark of the data requirement is achieved by repetitive virtual reference experiments (Fig. 1). The major considerable factors during data requirement setting are ability on validating and adapting performance, but also the cost and time for data collection when applicable. Likewise, the response data from the reference model can be provided with the noise(error) to have the data as realistic (with some technical data measurement error) from the real asset.

\subsection{Validation Metric Selection/Formulation}

Performance of the quantitative metric to assess the goodness-of-fit between the simulation output and real asset's response data is other major focus like roles of data in validation. While the nature of the simulation is known and the uncertainty types to assess are known, the metric selection/formulation will be easier. The nature of model in most of the application domains for their designated purposes is pre-known.

When performing model updating of complex structures, deterministic approaches are preferred [15] due to the associated computational efforts. Therefore, metrics performance evaluation and metric formulation are confined to the deterministic metrics at this phase of the project. The aleatory uncertainty assessment in the simulation model is outside the scope of this paper.

Multiple criterion types are available in literatures to validate the performance of a deterministic model. Among them, the magnitude discrepancy based and the correlation based are two wide categories of the metrics for deterministic validation. It is not possible to conclude that one criterion is better than another, as they assess different aspects on dataset. The choice of criteria to employ in validation should start with the features that are intended to be assessed [13].

The major features that can be assessed to determine the goodness-of-fit of prediction made with simulation are $[13,22]$

a. correlation/autocorrelation.

b. if the model output is unbiased with the observed data (e.g. comparing means).

c. the variance between observed and simulated data (i.e. magnitude discrepancy).

Determination of feature to be assessed takes the metric selection/formulation task a step ahead. When the variance is most considerable feature to track the goodness-of-fit of the model-output and the validation data, distance-based metrics (e.g. normalised mean square error, normalised mean absolute error, etc) are preferred. Similarly, for the case when correlation between the dataset is the feature to perform the validation, coefficient of correlation is preferred. Also, the possibility of implementation of a comprehensive metric combining both types can be explored when both categorical metrics are applicable. However, the feature determination could itself be challenging task, but it should be pre-established to accelerate the process. 
The role of validation metric formulation persists even after metric selection when multi-response data types available from simulation can be used for model's performance validation. Determining dependency of different data types during model performance validating is the further step of metric formulation in such cases.

Once the metric is formulated, it is further adopted as an objective function during optimisation-based model calibration. The best model's performance measuring metric during optimisation-based parameter updating is selected/formulated/generated using similar pre-mentioned repetitive virtual experiments by fixing the other influencing attributes.

\subsection{Performance Analysis on Model Convergence to Reference Model}

The validating data and metric requirement are recommended once convergence is confirmed not only with the basis of response data but also with the parameters value between the solution model reached and the reference model. The non-risk involved balance between the data and the cost involved on data collection and model optimisation with the data also identified by the series of virtual reference experimentation. Then, the recommended validating data and metric requirement are re-tested before to set as the benchmark with repeated reference experiments differing the initial state primary model and the reference model.

The overall approach can be implemented with automation within analytical platform provided into DT. Automation of the benchmarking approach supported with intelligence features anticipated within DT provides the roadmap for self-validation and adaptation of its simulation capability.

\section{CASE STUDY - CATHODIC PROTECTION ADAPTIVE MODEL PERFORMANCE VALIDATION USING PROPOSED APPROACH}

\subsection{Background}

Cathodic protection (CP) is most frequently used technique for protection of underground or underwater(seawater) metallic infrastructures from corrosion. The design of marine structures is typically based on design guidelines that specify the protection potentials on the structure to be achieved by the $\mathrm{CP}$ system. In the $\mathrm{CP}$ system, the rate at which elements of the protection system such as coatings to be assumed to degrade over the life of the structure. The performance of the $\mathrm{CP}$ system can be evaluated and optimised using a $\mathrm{CP}$ simulation model, which predicts year by year the protection potentials and the depletion of the anodes, and in the case of impressed-current-cathodic-protection, the current to be required by the system [21].

While this type of simulation provides valuable information to the design engineer by confirming that the required protection will be achieved. In reality, the actual performance of the CP system will be often different as coatings, for example, often degrade at different rates to that described in the design rules, environmental conditions may vary, the 'as-built' structure may be different and changes and retrofits are made over time. Integrating the $\mathrm{CP}$ data collected during the routine inspection surveys with a CP simulation model on calibrating/adapting the model to match the inspection data enables a 'DT' of the structure [23]. In this way, the simulation DT represents the behaviour of the structure and the CP system at the time the inspection survey was performed. This then provides the ability to predict the present and future protection for all parts of the structure. 
By repeating the process with each new inspection report, the engineer can monitor the differences between the model predictions and survey data systematically to assess current 'health' of the structure, identify anomalies, predict and plan for future risks, optimise the inspection strategy and provide early identification of problems that will require actions.

\subsection{Experimental Setup}

The applicability of the proposed approach is demonstrated with a simulator (BEASY)-based cathodic-protection modelling. The model works with determination of the distribution of electrical potential and protection current density on the electrode surfaces. It comes down on solving the well-known Laplace partial differential equation (1).

With assumption that electrolyte is homogeneous, the equation involved is given by

$$
-\nabla(k \nabla \varphi)=0
$$

where, $\mathrm{k}=$ electric conductivity, $\phi=$ electric potential and $\nabla$ is Nabla operator.

A CP simulation model of a marine structure (Figure 2) protected by sacrificial anodes is adopted. BEASY provides numerical approximation of the Laplace's equation for steadystate corrosion. Also, BEASY tool facilitates on geometrical modelling and meshing.

On having geometrical modelling, meshing and solver for numerical simulation, the next step on realisation of virtual replica of the physical CP system is calibration of the parametric model. Polarisation-related behaviour of the structure's materials and conductivity of the surrounding medium are the involved parameters. The size and shape-related parameters are

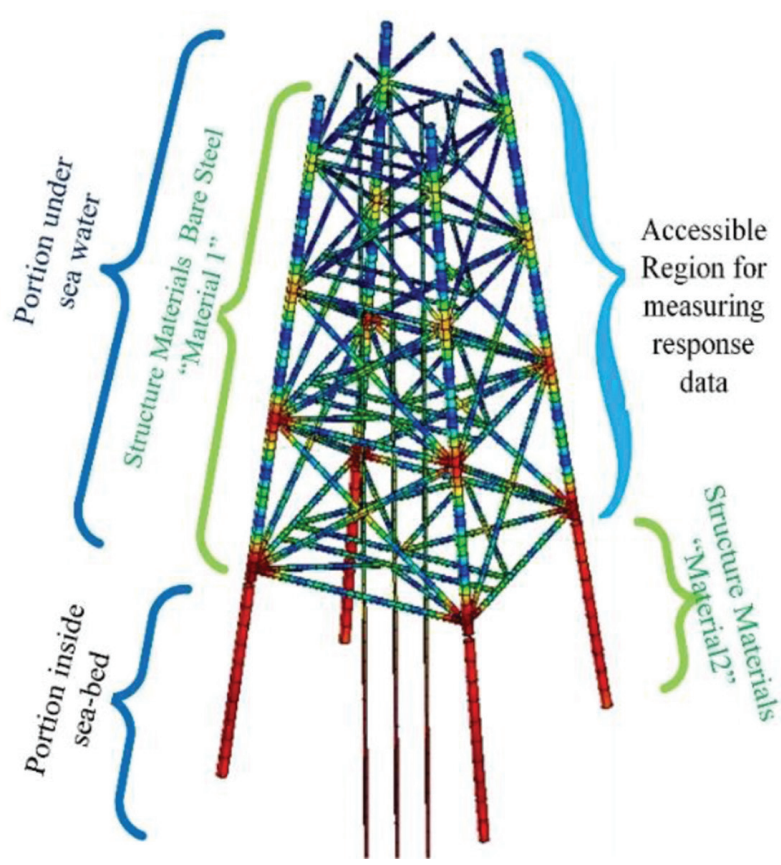

Figure 2: Marine structure on which adopted CP model is based upon. 
not within the scope of the research as such values can be obtained with the support of design data and/or provided structural measurements. Optimising the anode positions is the further task once the credibility of the model's prediction capability is achieved with the correctness of the input parameters.

The material properties-related parameters involved in the cathodic-protection model for the structure with sacrificial anodes (Fig. 2) are as follows:

$>$ Polarisation Behaviour: The relationship between potential and current density represents the electrode kinetics of the metal in the seawater. It provides the boundary condition while solving the numerical problem, that is, equation 1 .

* Polarisation curve for Material 1of structure (Fig. 2).

* Polarisation curve for Material 2 of structure (Fig. 2).

$>$ Conductivity/Resistivity: Surrounding Medium/Material related.

* Seawater-related conductivity (Siemen/m).

* Seabed-related conductivity (Siemen/m).

As the polarisation curves for representing the polarisation behaviour are graphical representation and dynamics with time, a quantitative representation should be established for re-adjustment of the polarisation data. To address the possible change in polarisation behaviour, the curve transformation value (expanding or squeezing factor) is taken as a variable (parameter) keeping the curve constant obtained from design rule (Fig. 3). This parameterisation concept can be understood as adapted Tafel slope [24] for polarisation behaviour of any materials involved. The transformation vector or parameter is termed as ' $p$-value' in this case study.

\subsubsection{Experimental Setup with Simulator Integration to the Analytical Platform}

Automation of the model validation and adaptation-related analytical task, that is, change of input parameters and analysis of the simulation performance, requires a supporting tool. When simulation model is developed using commercially available simulator, coupling of simulator tool and the supporting tool is implemented as self-sustaining approach.

MATLAB and MATLAB-based analytical [26] tools are used for the automation of the approach. The extensive data analysis, plotting capability and the availability of different optimisation algorithm of the MATLAB enables assess-modify-check loops to be completed in reduced computational time.

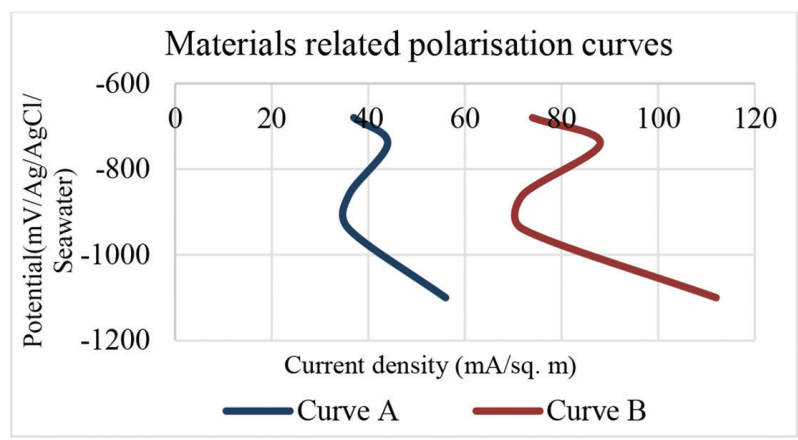

Figure 3: Two different polarisation curves that can be transformed from one to other with transformation factor ( $p$ value). 


\subsection{Approach Implementation on Benchmarking the Validation Data Requirement}

In this case study, the approach application for benchmarking response data requirements sufficiently enough to validate model's performance is demonstrated. The data types that can be practically obtained with measurement from the structure and simulation run as well are a) surface potential $(\mathrm{mV}), \mathrm{b})$ normal current density $\left(\mathrm{mA} / \mathrm{m}^{2}\right)$ and c) electric field $(\mathrm{mV} / \mathrm{m})[21]$.

While the approach is to find the benchmark of data requirements, other attributes involved are fixed beforehand. Normalised mean square difference with fixed weightage constant for multiple data types is fixed as the validating metric and as an objective function for optimisation. Similarly, for optimisation-based parameter updating, a gradient-based 'Newton-quasi' algorithm and MATLAB provided tool 'fminunc' [25] are chosen. The reason for choosing gradient based as opposed to other machine learning techniques, such as genetic algorithms or neural networks, is that the problem space is mostly monotonic.

\subsubsection{Virtual Models in the Reference Experiments}

Primary and reference models are generated using the adopted structure-related geometrical data and meshing. The parameter(s) values of the 'Reference model' provided are different from the one of the 'primary initial model' on every reference experiments.

It is easily obtained from sensitivity analysis that the parameters more sensitive to accessible response data (Fig. 2) are ' $p$-value of Material 1 related polarisation curve' and 'Seawater conductivity'. On the first stage, only highly sensitive parameters are considered keeping the other parameters' value constant. A representative example of primary initial and reference model's parameters is presented in Table 1 .

One of the validation-dependent response data types (surface potential) for CP model for the given CP system (Fig. 2) can be visualised from Figure 4 for both the virtual models (Table 1), with data from reference model represented as validating data.

A case study with initial data dependency as Case I (Table 2a and Figure 4a model output) is initiated for analysis with the reference experiments. In this case, while relying upon only on 'Surface Potential data', for model validation and adaptation, the likelihood to reach to the wrong solution during optimisation-based parameter updating was found. It was discovered on parameter comparison between solution model from Case I (Table $2 b$ ) and the reference model (Table 1). Then, while selecting next set of response data as model validation data, normal current density data was further added keeping the initial quantity same but with diversification (Case II). On similar comparison like before, result shows Case II favours over Case I in regard of data dependency. These two cases form the basis of benchmarking model validation data requirement. On performance evaluation with re-testing (3.4) with other generated reference experiments, the benchmark (Case II) of response data requirement is reached for validation and adaptation of the $\mathrm{CP}$ model.

The result shows diversification in data selection could minimise the risk converging to local minima during the validation and adaptation route from initial primary model to the virtual

Table 1: Parameter's value provided for the models in a reference experiment

\begin{tabular}{lll}
\hline & $\begin{array}{l}\text { 'Material 1'-related } \\
\text { polarisation curve pvalue }\end{array}$ & Seawater conductivity \\
\hline Primary Initial model & 1.5000 & 2.7500 \\
ReferenceModel & 2.0000 & 3.3333 \\
\hline
\end{tabular}



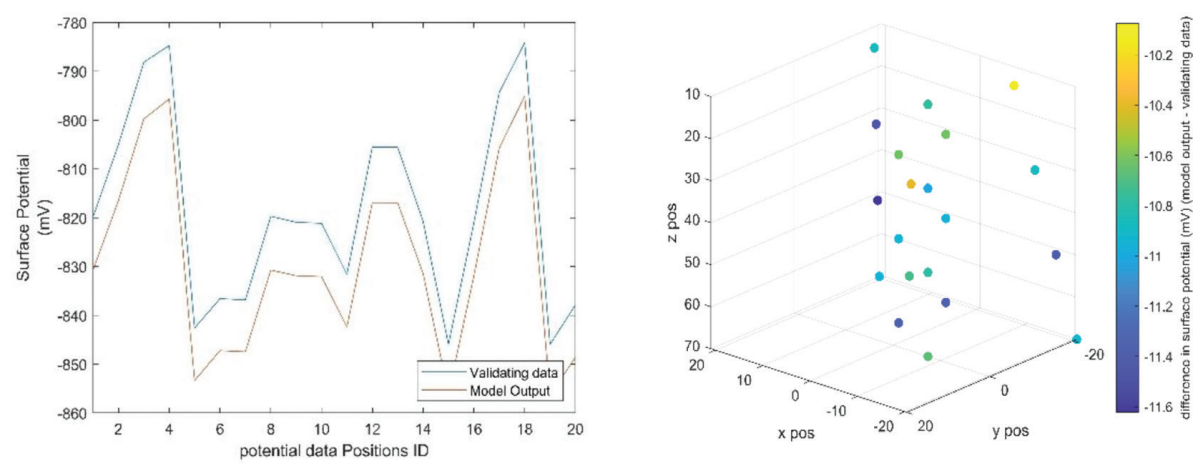

Figure 4: The graphical comparison between surface potential data from the simulation run for initial primary model (represented as model output) and from the reference model (as validating data) a) comparison of the response data with respect to position id b) difference shown at real 3D positions of the data.

reference model for the given case study. When relied upon data from Case I for model operational validation, one can reach the wrong assumption about the performance of the model.

The benchmarks provided might not only vary between models but also for same on different stages of operational lifespan due to change in integrity of the system. This recommends the integration of such benchmarking approach to the model with automation to support adaptation required at any instance of its operational lifespan. Similar approach can be implemented in metric formulation as the performance of the provided attributes differs.

Table 2: a) The validation data counts for two different experimental setups. b) Parameter's value in solution model reached for both cases.

(a)

Data counts

\begin{tabular}{lll}
\hline & Surface-potential $(\mathbf{m V})$ & Normal current density $(\mathbf{J})$ \\
\hline Case I & 20 & 0 \\
Case II & 15 & 5 \\
\hline
\end{tabular}

(b)

Parameter's value in solution model reached

\begin{tabular}{lll}
\hline & 'Material 1' polarisation p-value & Seawater conductivity \\
\hline Case I & 1.6055 & 2.6525 \\
Case II & 2.0099 & 3.3113 \\
\hline
\end{tabular}




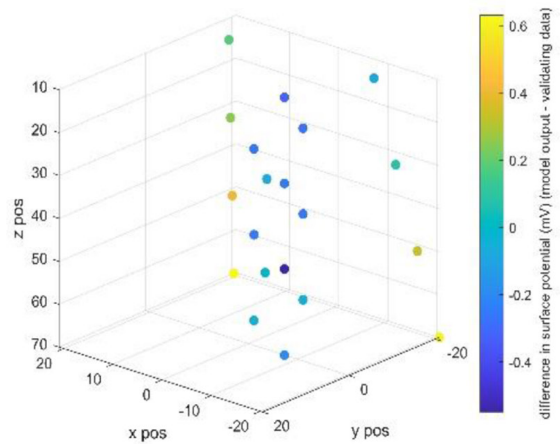

(a)

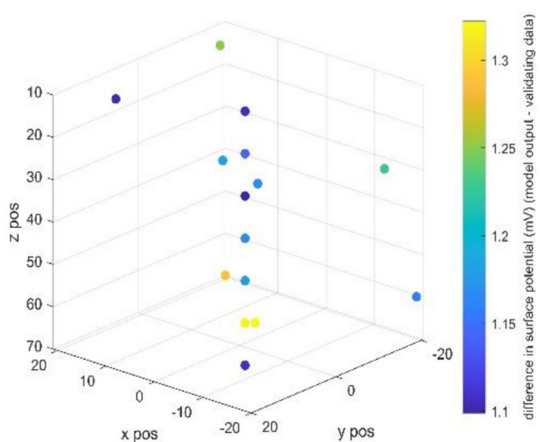

(b)

Figure 5: The discrepancies between solution model output and reference model output (validating data). a) Case I solution model and b) Case II solution model.

\section{CONCLUSION}

An approach is proposed to benchmark the data and the metric requirements before tailoring a parametric model to represent the real physical asset's behaviour. The approach utilises the virtual reference model and multiple virtual experiments before to reach the benchmark. Its applicability is demonstrated for benchmarking the quantity, quality and diversity of data requirements on validating the adopted $\mathrm{CP}$ model during its calibration. The demonstration of the approach with automation of the analytical task using a supporting tool suggests DT with model validation and adaptation framework incorporated within as a lifelong credible predictive tool for structural health assessment.

As a future work, the approach needs to be implemented in more complex model to have the benchmark of validation required on aligning route for its parametric calibration/adaptation. The approach implementation on reaching the benchmark of the operational validating metric also needs experimental demonstration. Also, the benchmark reached need to be applied to the real-world problem, that is, to calibrate the model based upon the response data from the physical CP system. The approach is, however, generic and not constrained to the corrosion and cathodic-protection domain.

\section{ACKNOWLEDGEMENTS}

This work has been undertaken as part of a match-funded $\mathrm{PhD}$ research between Computational Mechanics International Limited and Bournemouth University, UK.

\section{REFERENCES}

[1] Sargent, R.G., Verification and validation of simulation models. Journal of Simulation, 7(1), pp. 12-24, 2013. https://doi.org/10.1057/jos.2012.20

[2] Oliveira, H.L. \& Leonel, E.D., Constitutive relation error formalism applied to the solution of inverse problems using the BEM. Engineering Analysis with Boundary Elements, 108, pp. 30-40, 2019. https://doi.org/10.1016/j.enganabound.2019.07.023 
[3] Sehgal, S. \& Kumar, H., Structural dynamic model updating techniques: a state of the art review. Archives of Computational Methods in Engineering, 23(3), pp. 515-33, 2016. https://doi.org/10.1007/s11831-015-9150-3

[4] Liu, Y., Chen, W., Arendt, P. \& Huang, H.Z., Toward a better understanding of model validation metrics. Journal of Mechanical Design, 133(7), 2011. https://doi.org/ $10.1115 / 1.4004223$

[5] Pace, D.K., Modeling and simulation verification and validation challenges. Johns Hopkins APL Technical Digest, 25(2), pp. 163-72, 2004.

[6] Madni, A.M., Madni, C.C., \& Lucero, S., Leveraging digital twin technology in modelbased systems engineering. Systems, 7(1), p. 7, 2019. https://doi.org/10.3390/systems 7010007

[7] Wright, L., \& Davidson, S., How to tell the difference between a model and a digital twin. Advanced Modeling and Simulation in Engineering Sciences, 7(1), pp. 1-3, 2020. https://doi.org/10.1186/s40323-020-00147-4

[8] Barricelli, B.R., Casiraghi, E., \& Fogli, D., A survey on digital twin: Definitions, characteristics, applications, and design implications. IEEE Access, 14(7), pp. 167653-71, 2019. https://doi.org/10.1109/ACCESS.2019.2953499

[9] El Haouzi, H.B., Thomas, A., \& Charpentier, P., Toward adaptive modelling \& simulation for IMS: The Adaptive Capability Maturity Model and future challenges. IFAC Proceedings Volumes, 46(7), pp. 174-179, 2013. https://doi.org/10.3182/20130522-3br-4036.00104

[10]Brynjarsdóttir, J., \& O'Hagan, A., Learning about physical parameters: The importance of model discrepancy. Inverse Problems, 30(11), p. 114007, 2014. https://doi.org/ 10.1088/0266-5611/30/11/114007

[11] Higdon, D., Gattiker, J., Williams, B. \& Rightley, M., Computer model calibration using high-dimensional output. Journal of the American Statistical Association, 103(482), pp. 570-583, 2008. https://doi.org/10.1198/016214507000000888

[12] Sarin, H., Kokkolaras, M., Hulbert, G., Papalambros, P., Barbat, S. \& Yang, R. J., Comparing time histories for validation of simulation models: error measures and metrics. Journal of Dynamic Systems, Measurement, and Control, 132(6), 2010. https://doi.org/ $10.1115 / 1.4002478$

[13] Hora, J. \& Campos, P., A review of performance criteria to validate simulation models. Expert Systems, 32(5), pp. 578-595, 2015. https://doi.org/10.1111/exsy.12111

[14] Goller, B., Broggi, M., Calvi, A., \& Schuëller, G.I., A stochastic model updating technique for complex aerospace structures. Finite Elements in Analysis and Design, 47(7), pp. 739-752, 2011. https://doi.org/10.1016/j.finel.2011.02.005

[15] Glaessgen, E. \& Stargel, D., The digital twin paradigm for future NASA and US Air Force vehicles. In 53rd AIAA/ASME/ASCE/AHS/ASC structures, structural dynamics and materials conference 20th AIAA/ASME/AHS adaptive structures conference 14th AIAA (p. 1818), 2012.

[16] Ye, Y., Yang, Q., Yang, F., Huo, Y. \& Meng, S., Digital twin for the structural health management of reusable spacecraft: A case study. Engineering Fracture Mechanics, 234, p. 107076, 2020. https://doi.org/10.1016/j.engfracmech.2020.107076

[17] Fuller, A., Fan, Z., Day, C. \& Barlow, C., Digital twin: Enabling technologies, challenges and open research. IEEE Access, 8, p. 108952-71, 2020. https://doi.org/10.1109/ access. 2020.2998358 
[18] Gabor, T., Belzner, L., Kiermeier, M., Beck, M.T. \& Neitz, A., A simulation-based architecture for smart cyber-physical systems. In 2016 IEEE international conference on autonomic computing (ICAC) (pp. 374-379). IEEE, 2016.

[19] Park, B. \& Schneeberger, J.D., Microscopic simulation model calibration and validation: case study of VISSIM simulation model for a coordinated actuated signal system. Transportation Research Record, 1856(1), pp. 185-192, 2003. https://doi.org/ $10.3141 / 1856-20$

[20] Tahmasebi, F., Zach, R., Schuß, M., \& Mahdavi, A., Simulation model calibration: An optimization-based approach. In Proceedings of Fourth German-Austrian IBPSA Conference, BauSIM (pp. 386-391), 2012.

[21] Adey, R.A., Modelling of Cathodic Protection Systems. United Kingdom, WIT, 2006.

[22] Ni, D., Leonard, J.D., Guin, A. \& Williams, B.M., Systematic approach for validating traffic simulation models. Transportation Research Record, 1876(1), pp. 20-31, 2004.

[23] Adey, R., Peratta, C. \& Baynham, J., Corrosion Data Management Using 3D Visualisation and a Digital Twin. In NACE International Corrosion Conference Proceedings (pp. 1-13). NACE International, 2020.

[24] Kear, G. \& Walsh, F.C., The characteristics of a true Tafel slope. Corrosion and Materials, 30(6), pp. 51-55, 2005.

[25] Higham, D.J. \& Higham, N.J., MATLAB guide. Society for Industrial and Applied Mathematics, 2016. 